\title{
Evolutionary Models of RR Lyrae Stars
}

\author{
Young-Wook Lee \\ Department of Astronomy, Yale University, New Haven, CT, USA
}

\section{Introduction}

As tracers of old stellar populations and as primary Population II standard candles, RR Lyrae stars have played an important role in the development of modern astronomy. Our knowledge of stellar evolution has identified these variable stars in a core helium burning phase of low-mass star evolution, the horizontal-branch (HB) phase. Consequently, not only to understand fully the nature of RR Lyrae stars, but also to apply them correctly as population probes and distance indicators, we must understand the underlying evolutionary effect of HB stars.

In this paper, I briefly review the most important properties of RR Lyrae stars predicted from the HB evolutionary models, and present many pieces of supporting evidence for these models. For the implications of these models on the chronology of the Galactic formation and on the cosmological distance scale, the reader is referred to several recent publications by Lee (1992a,b,c,d).

\section{Properties of RR Lyrae Stars Predicted from HB Population Models}

The construction of HB population models is straightforward, once we have all the evolutionary tracks for $\mathrm{HB}$ stars of different masses for a given composition. It is generally assumed that the distribution of mass on the $\mathrm{HB}$ is Gaussian, resulting from variable amounts of mass-loss on the giant-branch. For each HB star, the time elapsed since the zero-age $\mathrm{HB}$ (ZAHB) is obtained by using a uniform random number generator. When the HB stars fall into the instability strip, we treat them as RR Lyrae variables. The HB population models yield the luminosity, mass, and effective temperature of each star within the instability strip; hence it is straightforward to calculate the periods of model RR Lyrae stars from the period-mean density relation (e.g., van Albada and Baker 1971). I refer the reader to Lee et al. (1990) and Lee (1990) for details of the model constructions.

As we have seen in this meeting, there is still some debate concerning the correlation between the luminosity of RR Lyraes and metallicity and other related problems, such as the Sandage period-shift effect. I believe that the reason for this annoying situation is nothing but due to the ignorance of the effect of post ZAHB evolution in some investigations. The importance of the effect of post ZAHB evolution on the properties of RR Lyrae stars is demonstrated in Figure 1, where we have two HB models of the same metallicity but different HB morphology. The model (a) is for the globular cluster like M3, which 


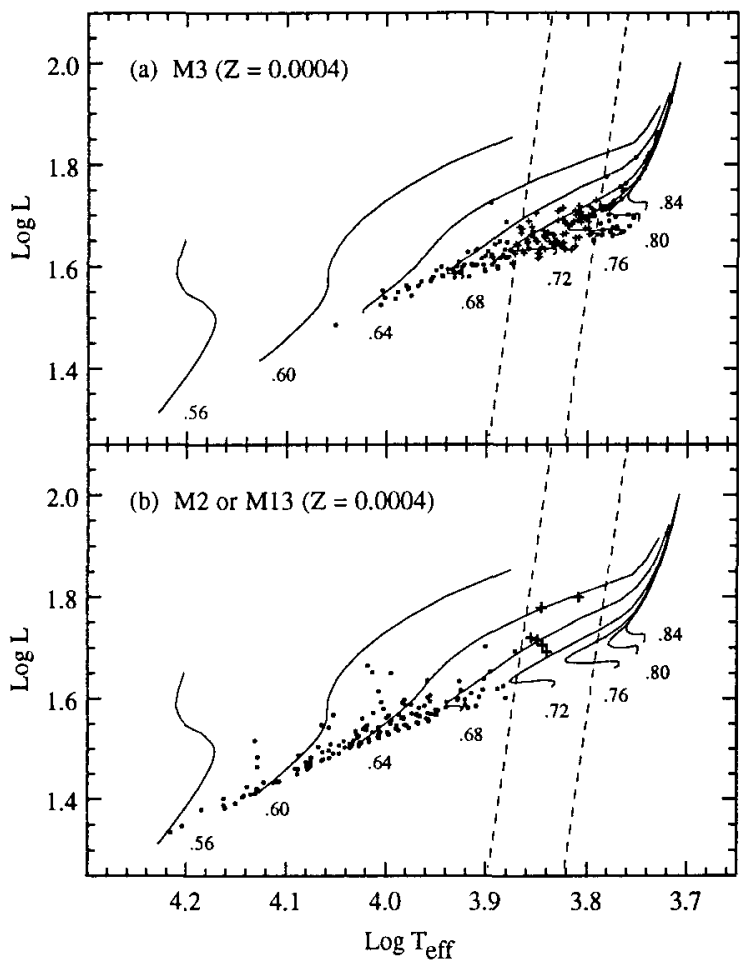

Fig. 1. The HB population models for M3 and M13 (or $M 2$ ) with HB evolutionary tracks $\left(Y_{H B} \approx 0.22\right)$. The instability strip is represented schematically by dashed lines, and each track is labeled by its total mass in solar units (from Fig. 4 of Lee 1990).

possesses nearly equal numbers of red and blue HB stars, while model (b) is for the globular cluster like M13 or M2, whose HB is almost entirely to the blue of the instability strip. We can see in model (a) that most RR Lyraes are near the ZAHB; hence the effect of evolution is relatively small. In model (b), however, almost all RR Lyraes are highly evolved stars from the blue HB, and thus the mean RR Lyrae luminosity $\left.\left(<\mathrm{M}_{\mathrm{v}}{ }^{\mathrm{RR}}\right\rangle\right)$ for model (b) is significantly brighter than that for model (a), even though they have the same metal abundance. Note also that the variables in model (b) are less massive than the ones in model (a).

Extensive model calculations by Lee $(1990,1992 b)$ demonstrate this effect more clearly. Figure 2 presents the results of such calculations for $\left\langle\mathrm{M}_{\mathrm{v}}{ }^{\mathrm{RR}}\right\rangle$, mean mass of $\mathrm{RR}$ Lyraes (<Mass $>$ ), and period-shift (at fixed $\mathrm{T}_{\text {eff }}$ ) as functions of my HB morphology index under various assumptions regarding the metal abundance. We can see here clearly that $\left\langle\mathrm{M}_{\mathrm{v}}{ }_{\mathrm{RR}}>\right.$ and $<$ Mass $>$ depends not only on metal abundance (as widely assumed) but also on HB morphology due to the effect of post ZAHB evolution. According to the period-mean density relation, the periods of variable stars are proportional to their luminosity, but are inversely proportional to their mass. Consequently, the period-shift of RR Lyraes 


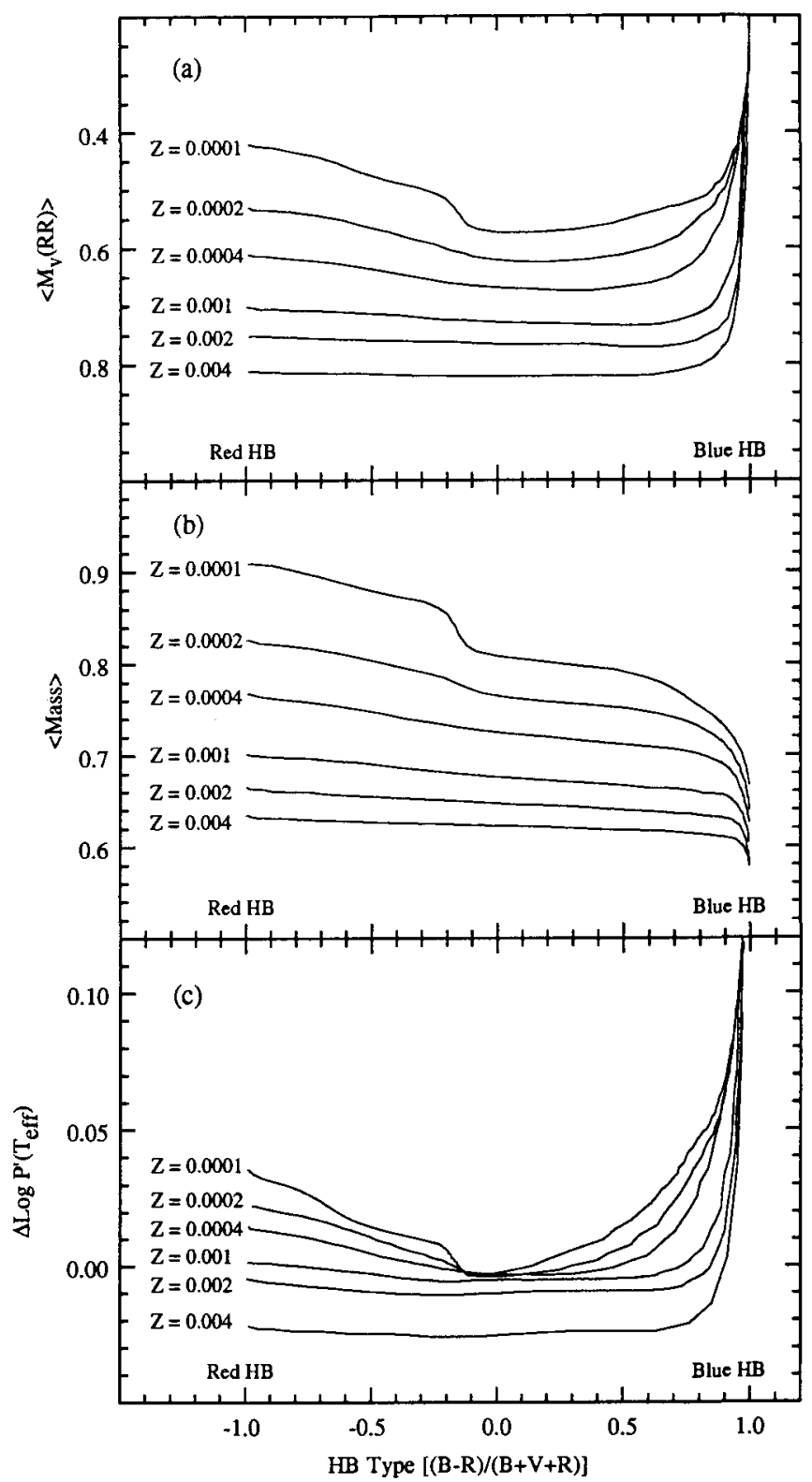

Fig. 2. The HB population model calculations for $\left\langle\mathrm{M}_{\mathrm{v}}{ }^{\mathrm{RR}}\right\rangle$, mean mass of RR Lyraes ( $<$ Mass $>$ ), and period-shift (at fixed $\left.\mathrm{T}_{\text {eff }}\right)$ as functions of $\mathrm{HB}$ type and metallicity $\left(\mathrm{Y}_{\mathrm{HB}} \approx 0.22, \sigma_{\mathrm{M}}=0.01 \mathrm{M}_{\odot}\right)$. The quantity $(B-R) /(B+V+R)$ is a new index devised by Lee (1989) to characterize the morphology of the HB (B, V, and $R$ are the numbers of blue $H B, R R$ Lyrae variable, and red $H B$ stars, respectively). 
measured at a given effective temperature $\left[\Delta \log \mathrm{P}^{\prime}\left(\mathrm{T}_{\mathrm{eff}}\right)=\log \mathrm{P}^{\prime}-\log \mathrm{P}_{\mathrm{M} 3}\right.$ at $\log \mathrm{T}_{\mathrm{eff}}=$ 3.83 ] is much more sensitive to the HB morphology (see Fig. 2c). Similarly, we can also calculate the mean period of type ab RR Lyraes as functions of HB type and metallicity, once some reasonable assumption is made regarding the transition temperature between the first overtone and fundamental mode of pulsation (see Lee and Zinn 1990 and below).

\section{Supporting Evidence}

Because of their impacts on the formation chronology of the Galaxy (see Lee 1992a,c) and on the cosmological distance scale (see Lee 1992b,d), it is of considerable importance to determine whether these new evolutionary models are correct. Below, I list many pieces of supporting evidence for these models.

\subsection{Period Changes of RR Lyrae Variables}

Since the periods of RR Lyrae variables should be either increasing, if the stars evolve from blue to red in the HR diagram, or decreasing, if they evolve from red to blue (Eddington 1918), the secular period changes of RR Lyrae stars provide a decisive test of the evolutionary models. If, as predicted by the Lee (1990) HB evolutionary models (see Fig. 1b), most RR Lyraes stars in blue HB clusters pass through the instability strip from blue to red toward the end of core helium burning phase, we expect large positive period changes for these RR Lyraes. In fact, Lee (1991a) has shown that the mean rate of period change in RR Lyrae stars depends sensitively on HB type due to the effect of post ZAHB evolution. In particular, he has shown that the observed period changes for the five beststudied clusters are consistent with those expected from the evolutionary models.

\subsection{The RR Lyrae Stars in the Globular Cluster $\omega$ Centauri}

As discussed above (see Fig. $2 \mathrm{a}$ ), the HB evolutionary models have shown that $\left\langle\mathrm{M}_{\mathbf{v}}{ }^{\mathrm{R}}{ }_{>}\right.$ depends sensitively on HB morphology as well as metallicity because of the effect of post ZAHB evolution. This has an important effect on the mean correlation between $\left\langle\mathrm{M}_{\mathrm{v}}{ }^{\mathrm{R}}{ }_{\rangle}\right.$ and metallicity among the Galactic globular cluster system, because the HB morphology itself varies with $[\mathrm{Fe} / \mathrm{H}]$. As illustrated in Figure 3, the observed $\mathrm{HB}$ type gets bluer with decreasing $[\mathrm{Fe} / \mathrm{H}]$, and in the mean, the clusters of intermediate metallicity $(-1.9<[\mathrm{Fe} / \mathrm{H}]$ $<-1.4$ ) have the bluest HBs (e.g., M2, M13, M22). But, as $[\mathrm{Fe} / \mathrm{H}]$ decreases still further, the HBs move back through the instability strip, producing the clusters with slightly redder HB types (e.g., NGC 5466, M53, M15). This implies that the HB morphology is not a monotonic function of [Fe/H] (Lee 1990, 1991b; see also Renzini 1983). When this effect is combined with the model calculations for $\left\langle M_{v} R>\right.$ in Fig. 2a, the models predict a mean correlation between $\left\langle\mathrm{M}_{\mathbf{v}}{ }^{\mathrm{RR}}>\right.$ and metallicity for the Galactic halo population that looks like a step function (see solid line in Fig. 4; see also Lee 1991b for details). 


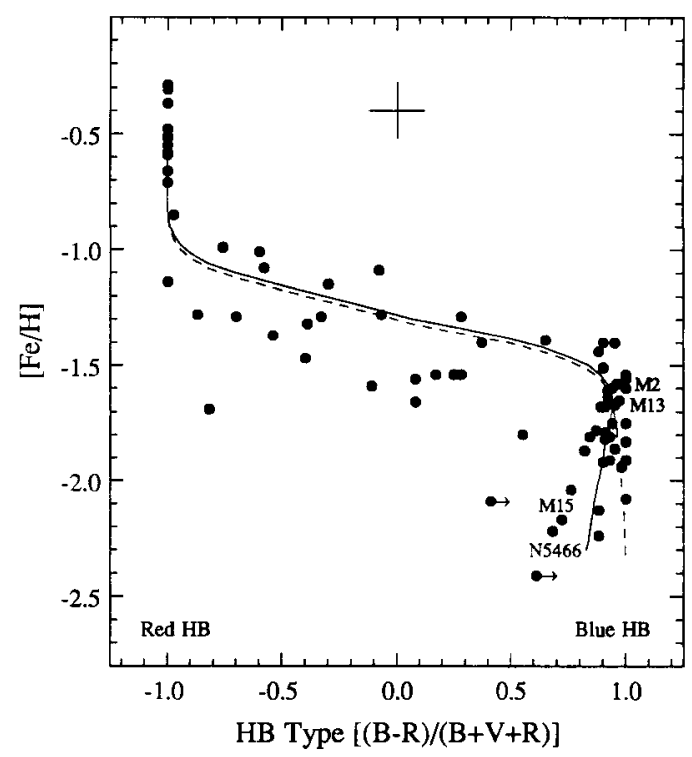

Fig. 3. HB type index is plotted against $[\mathrm{Fe} / \mathrm{H}]$ for Galactic globular clusters $\left(\mathrm{R}_{\mathrm{G}}<40 \mathrm{kpc}\right.$; from Lee 1991b). The solid line is a model locus with nonlinear mass-loss - [Fe/H] relation, and can be viewed as a mean correlation between $\mathrm{HB}$ type and $[\mathrm{Fe} / \mathrm{H}]$ for halo population. The dashed line is a model locus with fixed mass-loss, which produces too blue HB types for clusters having $[\mathrm{Fe} / \mathrm{H}]<-1.6$ (see Lee 1991b for details). This difference has profound effects on the values of $\left\langle\mathrm{M}_{b o l}{ }^{R R}\right\rangle,\left\langle\mathrm{P}_{a b}\right\rangle$, and period-shift (see solid and dashed lines in Figs. 4 and 6 for corresponding correlations in different diagrams).

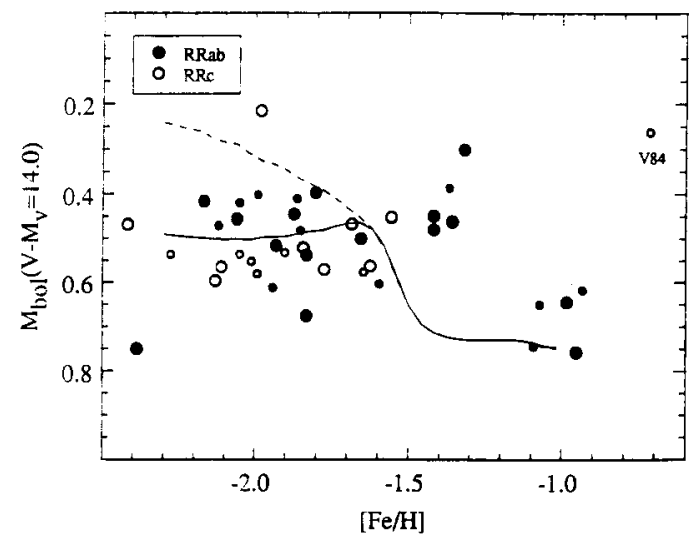

Fig. 4. Observed correlation between $\left\langle\mathrm{M}_{\text {bol }}{ }^{\mathrm{RR}}>\right.$ and $[\mathrm{Fe} / \mathrm{H}]$ for RR Lyrae stars in $\omega$ Centauri. The solid and dashed lines are model loci under two different assumptions regarding the $\mathrm{HB}$ type - $[\mathrm{Fe} / \mathrm{H}]$ correlations (see Fig. 3). Note that the correlation is not linear because $<\mathrm{M}_{\text {bol }}{ }^{\mathrm{RR}}>$ depends sensitively on HB morphology as well as metallicity (from Fig. 1 of Lee 1991b). 


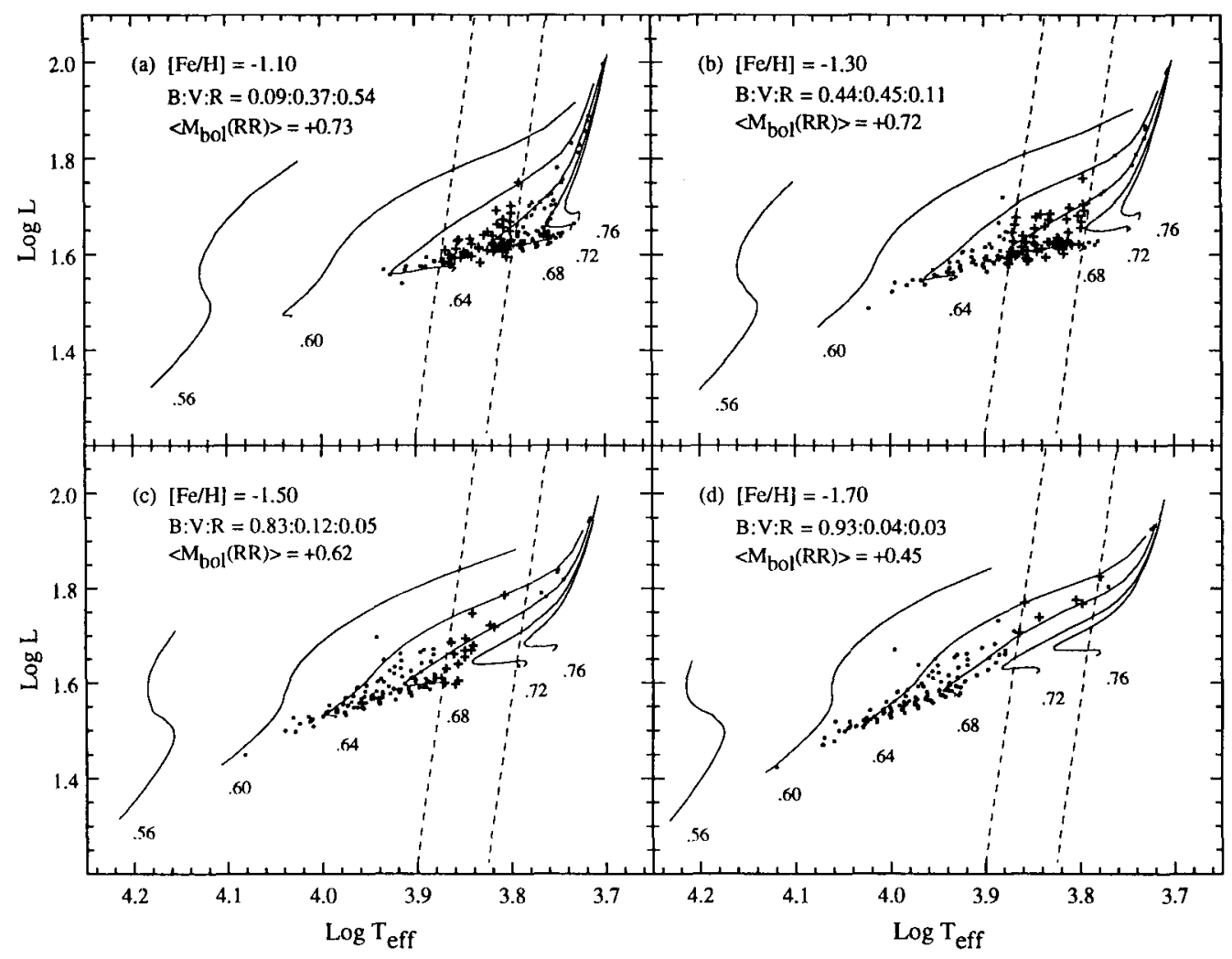

Fig. 5. Same as Fig. 1, but these models are constructed specifically to explain the origin of sudden upturn in $\left\langle\mathrm{M}_{\mathrm{bol}}{ }^{\mathrm{RR}}\right\rangle$ and $\left\langle\mathrm{P}_{\mathrm{w}}\right\rangle$ at $[\mathrm{Fe} / \mathrm{H}] \approx-1.5$ (see Figs. 4 and 6 ).

To test this model prediction, we need a large sample of RR Lyrae stars, spanning a large range of $[\mathrm{Fe} / \mathrm{H}]$, for which precise measurements of relative luminosity and $[\mathrm{Fe} / \mathrm{H}]$ exist. The RR Lyrae stars in $\omega$ Cen, which span a large range in $[\mathrm{Fe} / \mathrm{H}]$, are the most ideal sample for this test. In particular, the relative values of $M_{v}{ }^{R R}$ for $R R$ Lyraes in $\omega$ cen can be inferred straightforwardly from the apparent visual magnitudes because they are all located at the same distance and are all reddened by the same magnitudes. The observational data for the RR Lyraes in $\omega$ Cen are compared with the model prediction in Fig. 4, where one can see that the correlation becomes nonlinear at approximately $[\mathrm{Fe} / \mathrm{H}]=-1.5$, in reasonable agreement with the models.

The origin of this sudden upturn in $\left\langle\mathrm{M}_{\mathrm{v}}{ }^{\mathrm{RR}}>\right.$ at $[\mathrm{Fe} / \mathrm{H}] \approx-1.5$ is explained by a series of $\mathrm{HB}$ population models in Fig. 5, where one can see how sensitively the population of the instability strip changes with decreasing $[\mathrm{Fe} / \mathrm{H}]$. As $[\mathrm{Fe} / \mathrm{H}]$ decreases, $\mathrm{HB}$ morphology gets bluer, but between models (a) and (b), the increase in mean RR Lyrae luminosity is small because most RR Lyraes are still near the ZAHB (note that the evolution is slow near the ZAHB). However, as [Fe/H] decreases further, there is a certain point where the 
zero age portion of the HB just crosses the blue edge of the instability strip [see models (c) and (d)]. Then, only highly evolved stars from the blue HB can penetrate back into the instability strip, and the mean RR Lyrae luminosity increases abruptly. The increase in luminosity would continue if the HB morphology moves to blue monotonically with decreasing $[\mathrm{Fe} / \mathrm{H}]$ (see dashed line in Fig. 4). However, because of the nonmonotonic behavior of the $\mathrm{HB}$ morphology with decreasing $[\mathrm{Fe} / \mathrm{H}]$, the $\mathrm{HB}$ morphology gets slightly redder after model (d) and this effect offsets further increase in luminosity with decreasing $[\mathrm{Fe} / \mathrm{H}]$, producing the overall correlation that looks like a step function.

The fact that the correlation between $\left\langle M_{v}{ }^{R}>\right.$ and metallicity (and hence between period-shift and metallicity; see below) is not linear would clarify some of the disagreements with other investigators because fits of straight lines to different data sets produce significantly different slopes.

\subsection{The Oosterhoff Period Dichotomy among Globular Clusters}

The very same effect provides a natural explanation of the one of the long-standing problem in modern astronomy, the Oosterhoff (1939) period dichotomy among Galactic globular clusters. In Figure 6a, we plot the observed mean period of type ab RR Lyraes $\left(\left\langle\mathrm{P}_{\mathrm{ab}}\right\rangle\right)$ in globular clusters as a function of $[\mathrm{Fe} / \mathrm{H}]$. The solid line is a correlation predicted from the model calculations. Because the periods of RR Lyrae variables are proportional to their luminosity, it is not surprising to see again that the correlation looks like a step function. Intermediate values of $\left\langle\mathrm{P}_{\mathrm{ab}}\right\rangle$ between the two groups can be produced in principle, but only by clusters within a very narrow range of $\mathrm{HB}$ type and $[\mathrm{Fe} / \mathrm{H}]$ (see Lee and Zinn 1990). This explains why $\sim 50$ clusters in the Galaxy containing sufficient numbers of RR Lyrae variables fall into one or the other Oosterhoff group. Following the analyses of Lee et al. (1990), these calculations assume a small amount of hysteresis in mode switching, which is equivalent to a shift of $\sim 100 \mathrm{~K}$ in the $T_{\text {eff }}$ of the transition edge, depending on the direction of evolution. While this assumption is necessary if the models are to match the observed difference in $\left\langle\mathrm{P}_{\mathrm{ab}}\right\rangle$ between the Oosterhoff groups (see Lee et al. 1990), it is important to note that it cannot by itself produce the dichotomy.

Renzini (1983), Castellani (1983), and more recently Sandage (1990) have suggested that the Oosterhoff period dichotomy is due to the absence of RR Lyraes in clusters of intermediate metallicity caused by the nonmonotonic tracking of HB morphology with decreasing $[\mathrm{Fe} / \mathrm{H}]$. As noticed by Sandage (1990) himself, however, this cannot be the whole explanation because there are clusters on each side of the empty period range that have almost the same values of $[\mathrm{Fe} / \mathrm{H}]([\mathrm{Fe} / \mathrm{H}] \approx-1.5)$, yet have very different values of $\left\langle\mathrm{P}_{\mathrm{ab}}\right\rangle$. This sudden jump in $\left\langle\mathrm{P}_{\mathrm{ab}}\right\rangle$ at $[\mathrm{Fe} / \mathrm{H}] \approx-1.5$ is now understood as discussed above (see Fig. 5).

As first noticed by Lee (1990), the same effect is also seen in the correlation between the period-shift (at fixed $\mathrm{T}_{\text {eff }}$ ) and $[\mathrm{Fe} / \mathrm{H}]$ for field RR Lyrae stars (see Fig. 6b; see also Caputo and De Santis 1992). Again, the fact that the correlation between period-shift and metallicity is not linear would clarify some of the disagreements with other investigators because fits of straight lines to different data sets produce significantly different slopes. 


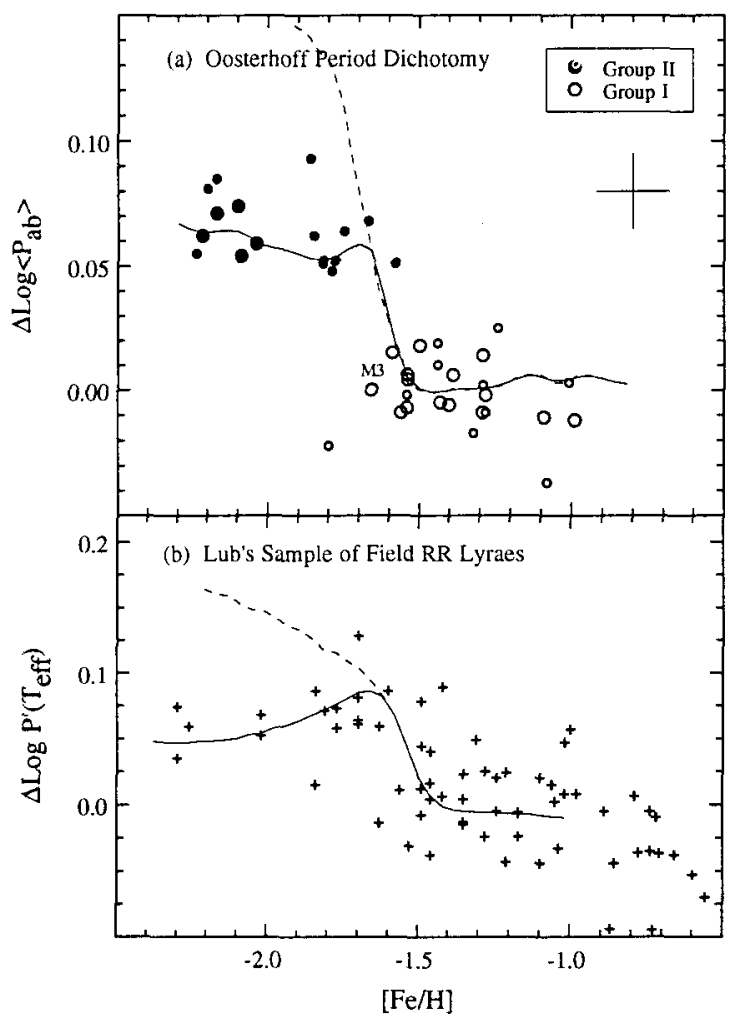

Fig. 6. (a) The Oosterhoff period dichotomy among Galactic globular clusters. (b) The correlation between period-shift (at fixed $\mathrm{T}_{\text {eff }}$ ) and metallicity for Lub's sample of field RR Lyrae stars. The solid and dashed lines are model loci (see Fig. 3 for corresponding model loci in HB type - $[\mathrm{Fe} / \mathrm{H}]$ plane). Note again that the correlations look like a step function (see text).

\subsection{Masses of the RR Lyrae Variables}

There has been some discrepancy between the masses of the double-mode RR Lyrae stars as determined from pulsation calculations and from stellar evolution models. The sense was that the masses derived from the Petersen diagram (pulsation calculations) are systematically lower than those indicated by the standard stellar evolution calculations by 0.10 $0.15 \mathrm{M}_{\circ}$ (see Lee et al. 1990). Sandage (1990), among others, considered this as evidence against the Lee et al. (1990) HB evolutionary models.

However, the most recent stellar pulsation calculations based on new Livermore OPAL opacities suggest that the double-mode masses are about $0.1-0.2 \mathrm{M}_{\odot}$ larger than those using earlier opacities (Cox 1991; Kovacs et al. 1991; see also Kovacs, this volume). To be consistent with the stellar pulsation calculations, we have used the same Livermore OPAL opacity tables in the calculation of HB evolutionary tracks (Yi, Lee, and Demarque 1992). The result is presented in Figure 7, where one can see that, unlike the 


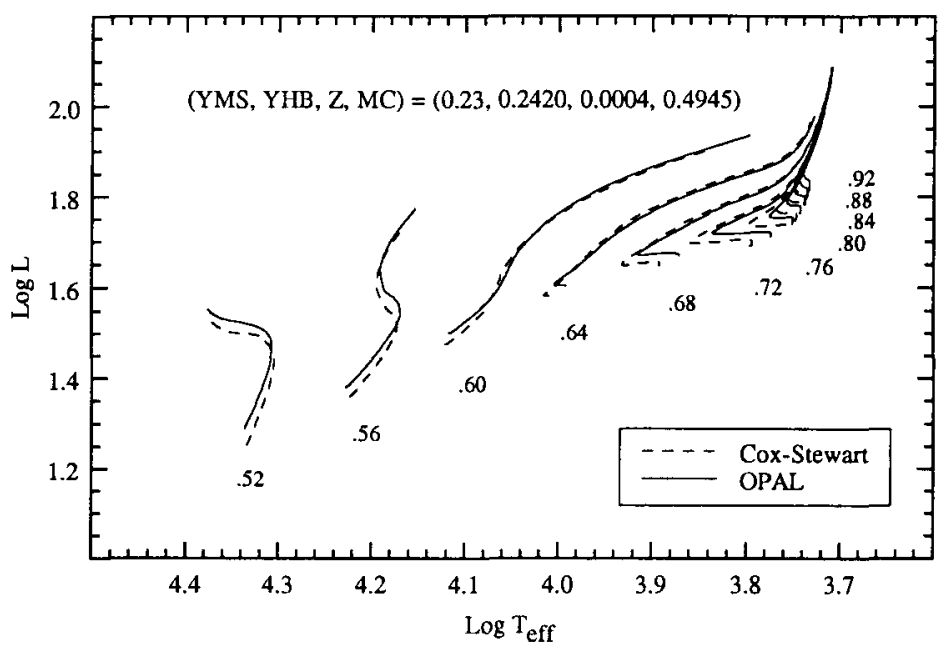

Fig. 7. The HB evolutionary tracks constructed with two opacity tables (from Yi, Lee, and Demarque 1992). Each track is labeled by its total mass in solar units.

pulsation calculations, the effect of new opacity is small in evolutionary calculations. In fact, the HB population models based on these tracks suggest that the decrease in mean $\mathrm{RR}$ Lyrae mass due to the new opacity is less than $0.01 \mathrm{M}_{\odot}$. Therefore, with the new Livermore OPAL opacities, both the stellar pulsation and evolution calculations predict approximately the same masses for the RR Lyrae stars. This is a strong independent check on the evolutionary models.

\subsection{Baade-Wesselink Measurements of Field RR Lyrae Stars}

One of the strongest observational evidence in favor of the HB evolutionary models is provided by three recent investigations of field RR Lyrae variables using the BaadeWesselink method (Cacciari et al. 1989; Liu and Janes 1990; Carney et al. 1992). These observations have yielded a relationship between $\left\langle\mathrm{M}_{\mathrm{v}}{ }^{\mathrm{RR}}>\right.$ and metallicity that has approximately one-half the slope of Sandage's (1982) relation (see also Sandage and Cacciari 1990), but is in excellent agreement with the Lee et al. (1990) HB evolutionary models. It is important to note that the analysis of Lee et al. (1990) did not include the extremely blue HB clusters of intermediate metallicity because in general they contain fewer variables. Consequently, their $\left\langle\mathrm{M}_{\mathrm{v}}{ }^{\mathrm{RR}}\right\rangle$ - metallicity correlation appears to be linear (see Fig. 10 of Lee et al. 1990). The sample of RR Lyrae stars used in the recent Baade-Wesselink analysis also lacks such highly evolved stars of intermediate metallicity, and therefore it is not surprising to see the excellent agreement between these two results. Clearly, it is of great importance to continue the Baade-Wesselink measurements of RR Lyrae stars in this metallicity range to see whether the correlation becomes nonlinear due to the effect of evolution (as is the case in the $\omega$ Cen). Note that Clementini and Cacciari (1990) already re- 
port two such highly evolved stars in this metallicity range (see also Cacciari, Clementini, and Fernley 1992).

Support for this work was provided by NASA through a grant HF-1014.01-90A awarded by the Space Telescope Science Institute, which is operated by the Association of Universities for Research in Astronomy, Inc., for NASA under contract NAS5-26555.

\section{References}

Cacciari, C., Clementini, G., and Fernley, J. A. 1992, ApJ, in press

Cacciari, C., Clementini, G., Prevot, L., and Buser, R. 1989, A\&A, 209, 141

Caputo, F., and De Santis, R. 1992, AJ, 104, 253

Carney, B. W., Storm, J., and Jones, R. V. 1992, ApJ, 386, 663

Castellani, V. 1983, Mem. Soc. Astron. Italiana, 54, 141

Clementini, G., and Cacciari, C. 1990, in Confrontation between Stellar Pulsation and Evolution, ed. C. Cacciari (San Francisco: ASP), p. 109

Cox, A. N. 1991, ApJL, 381, L71

Eddington, A. S. 1918, MNRAS, 79, 2

Kovacs, G., Buchler, J. R., and Marom, A. 1991, A\&A, 252, L27

Lee, Y.-W. 1989, Ph.D. thesis, Yale University

Lee, Y.-W. 1990, ApJ, 363, 159

Lee, Y.-W. 1991a, ApJ, 367, 524

Lee, Y.-W. 1991b, ApJL, 373, L43

Lee, Y.-W. 1992a, PASP, in press (September)

Lee, Y.-W. 1992b, Mem. Soc. Astron. Italiana, in press

Lee, Y.-W. 1992c, AJ, in press (November)

Lee, Y.-W. 1992d, in Variable Stars and Galaxies, ed. B. Warner (San Francisco: ASP), in press

Lee, Y.-W., Demarque, P., and Zinn, R. 1990, ApJ, 350, 155

Lee, Y.-W., and Zinn. R., 1990, in Confrontation between Stellar Pulsation and Evolution, ed. C.

Cacciari (San Francisco: ASP), p. 26

Liu, T., and Janes, K. 1990, ApJ, 354, 273

Oosterhoff, P. Th. 1939, Observatory, 62, 104

Renzini, A. 1983, Mem. Soc. Astron. Italiana, 54, 335

Sandage, A. 1982, ApJ, 252, 553

Sandage, A. 1990, ApJ, 350, 631

Sandage, A., and Cacciari, C. 1990, ApJ, 350, 645

van Albada, T. S., and Baker, N. 1971, ApJ, 169, 311

Yi, S., Lee, Y.-W., and Demarque, P. 1992, in preparation 